\title{
Transition Temperature of Lead-Free Piezoelectric Ceramics by Electrochemical Impedance Spectroscopy
}

\author{
A.M. Torres-Huerta, ${ }^{1, *}$ J.G. González-Reyes, ${ }^{2}$ M.E. Villafuerte-Castrejón, ${ }^{2}$ \\ F. González, ${ }^{2}$ E. Ramírez-Meneses, ${ }^{1}$ M.A. Domínguez-Crespo ${ }^{1}$ \\ ${ }^{1}$ Instituto Politécnico Nacional, CICATA-Altamira, Km 14,5 Carretera Tampico-Puerto \\ Industrial Altamira, Altamira, Tamps., México 89600 \\ ${ }^{2}$ Universidad Nacional Autónoma de México, Instituto de Investigaciones en Materiales, \\ Circuito Exterior s/n, Ciudad Universitaria, México, D. F. 04510 México
}

Received 25 April 2008; accepted 7 January 2009

\begin{abstract}
Piezoelectric ceramics with perovskite structure based on lead zirconate titanate (PZT) show excellent electrical properties near the morphotropic phase boundary (MPB) and thus are widely used for actuators, sensors as well as microelectronic devices. However, because of the high toxicity of lead oxide and its high vapour pressure during sintering, the use of lead-based ceramics has caused serious lead pollution and environmental problems; for this reason, the development of lead-free piezoelectric ceramics has been demanded to replace lead-based ceramics. Therefore, it is necessary to develop lead-free piezoelectric ceramics with good properties. Among the lead-free piezoelectric materials, the alkaline niobate-based perovskite compounds and Bi-containing materials, have attracted a large amount of attention because of their superior characteristics. The alkaline niobates $(\mathrm{K}, \mathrm{Na}) \mathrm{NbO}_{3}$ (KNN)-based material exhibits especially good piezoelectric properties and have been studied as substitutes for PZT piezoelectrics. In this work, alkaline niobate-based piezoelectric ceramics were sintetized, system $\left(\mathrm{K}_{0.5} \mathrm{Na}_{0.5}\right)_{\mathrm{x}} \mathrm{Li}_{(1-\mathrm{x})} \mathrm{Nb}_{\mathrm{y}} \mathrm{Ta}_{(1-\mathrm{y})} \mathrm{O}_{3}$, and were characterized by $\mathrm{X}$-ray diffraction (DRX) and scanning electron microscopy (SEM). In order to determine their transition temperature, electrochemical impedance spectroscopy (EIS) was used, and it was found the transition temperature was about $420{ }^{\circ} \mathrm{C}$, which is a desirable value in this kind of materials.
\end{abstract}

Keywords: PZT, alkaline niobate, transition temperature, piezoelectric ceramics, leadfree ceramics.

\footnotetext{
*Corresponding author. E-mail address: atohuer@ hotmail.com
} 


\section{Introducción}

Los materiales piezoeléctricos se utilizan en los transductores, dispositivos que convierten la energía eléctrica en esfuerzos mecánicos, o viceversa. Las aplicaciones comunes de los piezoeléctricos incluyen micrófonos, generadores ultrasónicos, sensores de presión y sonares. Entre este tipo de materiales se encuentran los titanatos de bario y plomo, zirconato de plomo $\left(\mathrm{PbZrO}_{3}\right)$, titanato zirconato de plomo (PZT), fosfato diácido de amonio $\left(\mathrm{NH}_{4} \mathrm{H}_{2} \mathrm{PO}_{4}\right)$ y cuarzo. Esta propiedad es característica de materiales que tienen estructuras cristalinas complicadas, con bajo grado de simetría. El comportamiento piezoeléctrico de una muestra puede mejorarse al calentarla por encima de la temperatura de Curie y enfriar después hasta temperatura ambiente en un campo eléctrico intenso (polarización) [1].

Los cerámicos piezoeléctricos, representados por el PZT son muy usados para actuadores, sensores y transductores, debido a sus excelentes propiedades piezoeléctricas. Sin embargo, la volatilización del $\mathrm{PbO}$ durante la sinterización a alta temperatura, no sólo provoca contaminación ambiental sino que también genera inestabilidad de la composición y de las propiedades eléctricas de los productos. Por ello, el desarrollo de cerámicos piezoeléctricos libres de plomo, para reemplazar los cerámicos base PZT, se ha convertido en la tendencia principal en el desarrollo actual de los materiales piezoeléctricos [2].

En la búsqueda de nuevos materiales, se ha encontrado que el titanato de bismuto y sodio, $\mathrm{Na}_{0,5} \mathrm{Bi}_{0,5} \mathrm{TiO}_{3}$ (NBT), es un buen candidato a cerámico piezoeléctrico libre de plomo, por su ferroelectricidad con una polarización remanente alta. Sin embargo, tiene la desventaja de poseer un alto campo coercitivo y alta conductividad, lo que causa problemas al polarizar. Por esta razón, se han estado investigando cerámicos que puedan polarizarse con facilidad. De estas investigaciones, se sabe que el titanato de bario, $\mathrm{BaTiO}_{3}(\mathrm{BT})$, y el titanato bismuto de potasio, $\mathrm{K}_{0,5} \mathrm{Bi}_{0,5} \mathrm{TiO}_{3}(\mathrm{KBT})$, materiales con fase tetragonal y los sistemas binarios NBT-BT y NBT-KBT, presentan buenas propiedades piezoeléctricas y dieléctricas en la composición cercana al límite de fase morfotrópica (MPB). Algunos informes recientes han demostrado la obtención de propiedades piezoeléctricas preferenciales al añadir KBT y BT al NBT para formar el sistema ternario NBT-BT-KBT. Sin embargo, las propiedades piezoeléctricas, dieléctricas y composición MPB todavía no están muy aclaradas. Li et al., reportaron las propiedades piezoeléctricas y dieléctricas, además de investigar el intervalo de composición del MPB para el sistema ternario (13x)NBT-2xKBT-xBT, manteniendo constante la relación KBT y BT. Por otra parte, los niobatos alcalinos han sido estudiados con renovado interés, debido a que representan una alternativa menos contaminante para sustituir a los cerámicos piezoeléctricos en base plomo y presentan, además, buenas propiedades piezoeléctricas y ferroeléctricas. Una de las desventajas de estos niobatos alcalinos es que presentan una baja sinterabilidad [2-3].

Por ello, el objetivo de este trabajo fue la obtención de cerámicos piezoeléctricos libres de plomo, de base niobatos alcalinos, de acuerdo al sistema $\left(\mathrm{K}_{0,5} \mathrm{Na}_{0,5}\right)_{\mathrm{x}} \mathrm{Li}_{(1-}$ ${ }_{\mathrm{x})} \mathrm{Nb}_{\mathrm{y}} \mathrm{Ta}_{(1-\mathrm{y})} \mathrm{O}_{3}$, con una sinterización adecuada. Estos materiales se caracterizaron estructural y morfológicamente mediante difracción de rayos X y microscopía 
electrónica de barrido. Para determinar la temperatura de transición se utilizó espectroscopia de impedancia electroquímica, encontrándose que esta temperatura está alrededor de $420{ }^{\circ} \mathrm{C}$, valor deseable en este tipo de materiales.

\section{Método experimental}

Se sintetizaron cerámicos $\left(\mathrm{K}_{0,5} \mathrm{Na}_{0,5}\right)_{0,95} \mathrm{Li}_{0,05} \mathrm{Nb}_{\mathrm{y}} \mathrm{Ta}_{(1-\mathrm{y})} \mathrm{O}_{3} \quad(\mathrm{y}=0,5 ; 0,7 ; 0,8)$ mediante el método convencional de reacciones en estado sólido, utilizando polvos de alta pureza $(99,9 \%)$ de $\mathrm{Na}_{2} \mathrm{CO}_{3}, \mathrm{Li}_{2} \mathrm{CO}_{3}, \mathrm{~K}_{2} \mathrm{CO}_{3}, \mathrm{Nb}_{2} \mathrm{O}_{5}$ y $\mathrm{Ta}_{2} \mathrm{O}_{5}$. Los polvos se pesaron en cantidades estequiométricas y se molieron durante 24 horas con acetona como disolvente. Las mezclas obtenidas se calcinaron a $700{ }^{\circ} \mathrm{C}$ durante 2 horas y nuevamente se molieron otras 24 horas en acetona. Las mezclas resultantes se compactaron y se sinterizaron a temperaturas de 900 a $1200{ }^{\circ} \mathrm{C}$ para obtener cerámicos densos. La caracterización estructural de las muestras preparadas se realizó por difracción de rayos X (DRX, D8-Advance, Bruker Instruments) con radiación $\mathrm{CuK} \alpha$ a $40 \mathrm{kV}$ y $35 \mathrm{~mA}$. Las medidas se realizaron a temperatura ambiente en un intervalo $2 \theta$ de $3^{\circ}-120^{\circ}$ con un paso de $0,02^{\circ}$ y velocidad de barrido de $8,5 \mathrm{~s} /$ paso. Los datos experimentales de DRX de las muestras se refinaron mediante el análisis Rietveld. La caracterización morfológica se realizó mediante microscopia electrónica de barrido (MEB). Para las pruebas de impedancia electroquímica, las caras de las pastillas se recubrieron con tintura de plata y se dejaron a temperatura ambiente para evaporar los disolventes presentes en la tintura; la plata realizó la función de electrodo. Las pruebas de impedancia electroquímica se realizaron en un equipo Agilent 4294A con interfase para alta temperatura, incrementando poco a poco la temperatura; inicialmente, el incremento fue de $25^{\circ} \mathrm{C}$ y se registraba la medida correspondiente; a partir de $\operatorname{los} 200{ }^{\circ} \mathrm{C}$, el incremento fue de $10{ }^{\circ} \mathrm{C}$, con la finalidad de determinar la temperatura de Curie del material.

\section{Resultados y análisis}

\section{Caracterización morfológica y estructural}

La Fig. 1 muestra los difractogramas obtenidos para las diferentes fases de cerámicos piezoeléctricos libres de plomo. Estos resultados concuerdan con los reportados por Zuo et al. [4] y Matsubara et al. [5], quienes trabajaron niobatos alcalinos y, de acuerdo a los análisis Rietveld realizados a las muestras del presente trabajo, la fase obtenida es la ortorrómbica.

En las Fig. 2-4 se muestran las imágenes de los materiales con composiciones $\mathrm{KNL}-\mathrm{Nb}_{\mathrm{x}} \mathrm{Ta}_{(1-\mathrm{x})} \mathrm{O}_{3}$, las cuales fueron sinterizadas a diferentes temperaturas. Se observa la variación en el tamaño de cristal de una composición a otra $y$, contrariamente a lo señalado para otros sistemas cercanos $\left(\left(\mathrm{Na}_{0,5} \mathrm{~K}_{0,5}\right)\left(\mathrm{Nb}_{1 \text { - }}\right.\right.$ $\left.{ }_{\mathrm{x}} \mathrm{Ta}_{\mathrm{x}}\right) \mathrm{O}_{3}$ por Matsubara et al. [5]), quienes han señalado que conforme aumenta el contenido de Ta, el tamaño de grano disminuye, en nuestro sistema este comportamiento no se aprecia claramente, ya que se observan granos de mayor tamaño en la muestra KNL-Nb $\mathrm{Ka}_{70} \mathrm{Ta}_{30} \mathrm{O}_{3}$ que en las otras dos (KNL-Nb $\mathrm{Kb}_{50} \mathrm{Ta}_{50} \mathrm{O}_{3} \mathrm{y}$ $\mathrm{KNL}-\mathrm{Nb}_{80} \mathrm{Ta}_{20} \mathrm{O}_{3}$ ). La distribución de los granos es uniforme en las muestras 
KNL-Nb ${ }_{50} \mathrm{Ta}_{50} \mathrm{O}_{3}$ y KNL-Nb $\mathrm{Kb}_{80} \mathrm{Ta}_{20} \mathrm{O}_{3}$, no así en la muestra KNL-Nb $\mathrm{Nb}_{70} \mathrm{Ta}_{30} \mathrm{O}_{3}$, en la cual se observa un crecimiento anormal de los granos.

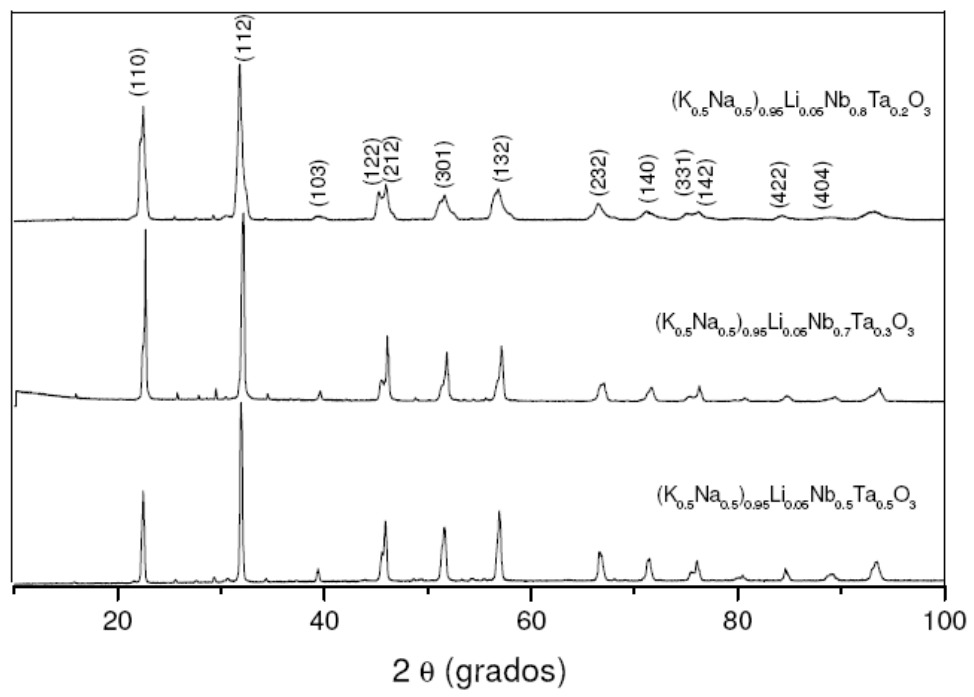

Figura 1. Patrones de difracción de $\left(\mathrm{K}_{0,5} \mathrm{Na}_{0,5}\right)_{0,95} \mathrm{Li}_{0,05} \mathrm{Nb}_{\mathrm{y}} \mathrm{Ta}_{(1-\mathrm{y})} \mathrm{O}_{3}$; a) y $=0,5$; b) y $=$ 0,7 ;) $\mathrm{y}=0,8$.

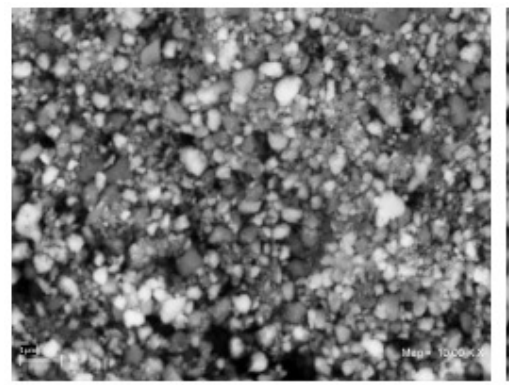

(a)

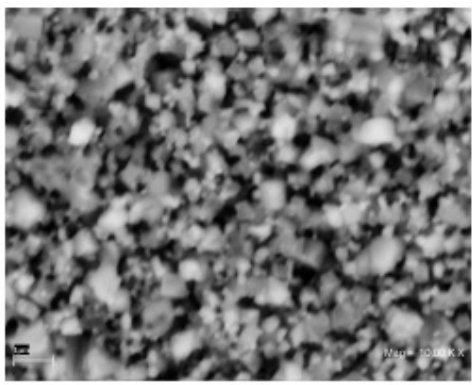

(b)

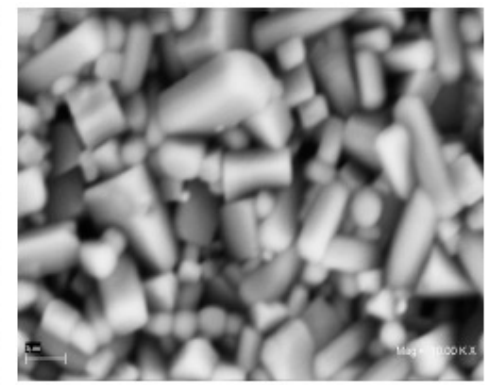

(c)

Figura 2. KNL-Nb $\mathrm{Nb}_{50} \mathrm{Ta}_{50} \mathrm{O}_{3}$ (a) Pastilla antes de sinterizar (b) Pastilla sinterizada a 1100 ${ }^{\circ} \mathrm{C}(\mathbf{1 0 0 0 0} \mathrm{X})$ (c) Pastilla sinterizada a $1200{ }^{\circ} \mathrm{C}(\mathbf{1 0 0 0 0} \mathbf{x})$.

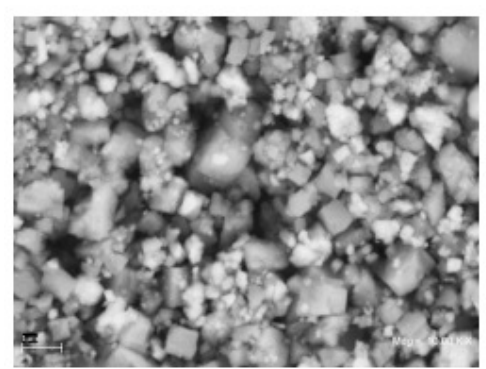

(a)

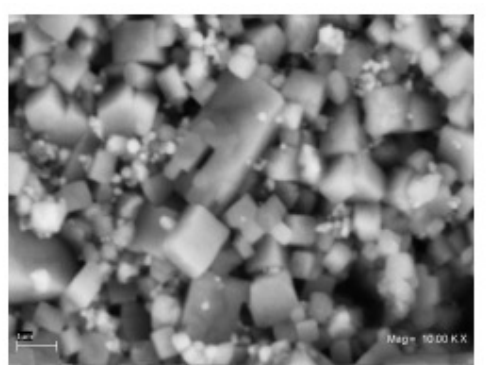

(b)

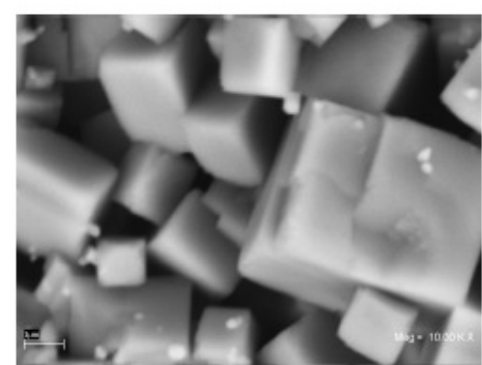

(c)

Figura 3. $\mathrm{KNL}-\mathrm{Nb}_{70} \mathrm{Ta}_{30} \mathrm{O}_{3}$ (a) Pastilla antes de sinterizar (b) Pastilla sinterizada a 1100 ${ }^{\circ} \mathrm{C}(\mathbf{1 0 0 0 0} \mathbf{x})$ (c) Pastilla sinterizada a $1200{ }^{\circ} \mathrm{C}(\mathbf{1 0 0 0 0} \mathbf{x})$. 


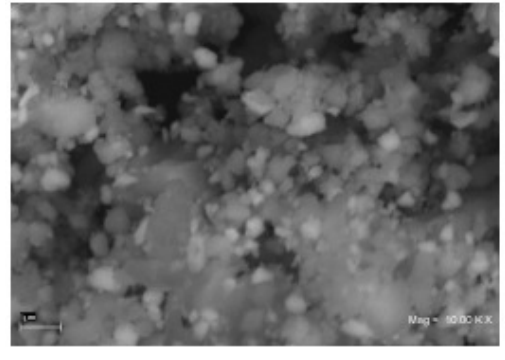

(a)

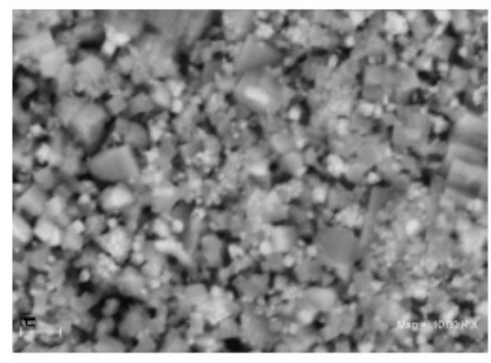

(b)

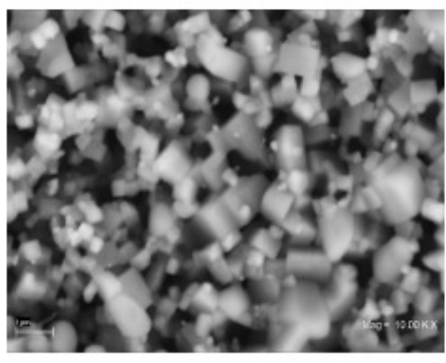

(c)

Figura 4. $\mathrm{KNL}-\mathrm{Nb}_{80} \mathrm{Ta}_{20} \mathrm{O}_{3}$ (a) Polvos sin sinterizar, (10000 x) (b) Pastilla sinterizada a $1000{ }^{\circ} \mathrm{C}(\mathbf{1 0 0 0 0} \mathbf{x})(\mathrm{c})$ Pastilla sinterizada a $1100{ }^{\circ} \mathrm{C}(\mathbf{1 0 0 0 0} \mathbf{x})$.

\section{Medidas electroquímicas}

La espectroscopia de impedancia electroquímica es una técnica muy útil para la caracterización de materiales cerámicos. La mayor parte de los parámetros eléctricos de un material se puede determinar a partir de los valores de impedancia compleja del mismo, tomando lecturas cada 8 a $10{ }^{\circ} \mathrm{C}$, desde la temperatura ambiente hasta $100{ }^{\circ} \mathrm{C}$ después de la temperatura de transición, aproximadamente. Posteriormente, se toman los datos correspondientes a distintas frecuencias (en este caso, a 1, 10 y $100 \mathrm{kHz}$ ). Considerando el factor geométrico (esto es, dimensiones de la pastilla) y mediante el tratamiento matemático de la relación entre la impedancia y la capacitancia, se llega a una expresión de la permitividad relativa, $\varepsilon$ ', en función de la frecuencia, $\omega$, impedancia real Z' e impedancia imaginaria $Z$ '', de acuerdo a las siguientes expresiones:

$$
\mathrm{Z}(\omega)=\mathrm{Z}^{\prime}+i \mathrm{Z}^{\prime \prime}
$$

$\mathrm{y}$

$$
Y=\frac{\mathrm{Z}^{\prime}-i \mathrm{Z}^{\prime \prime}}{\mathrm{Z}^{\prime 2}+\mathrm{Z}^{\prime 2}}
$$

también,

$$
Y=i \omega C \varepsilon^{\prime}+\omega C \varepsilon^{\prime \prime}
$$

De las ecuaciones (2) y (3) se tiene que:

$$
\omega C \varepsilon^{\prime \prime}+i \omega C \varepsilon^{\prime}=\frac{Z^{\prime}-i Z^{\prime \prime}}{Z^{\prime 2}+Z^{\prime \prime 2}}
$$

Arreglando la ecuación anterior:

$$
\varepsilon^{\prime}=-\frac{Z^{\prime \prime}}{\omega C\left(Z^{\prime 2}+Z^{\prime \prime 2}\right)}
$$


Por último, considerando que $C=\varepsilon_{0} \frac{A}{l}$, donde $\mathrm{C}$ es la capacitancia, $A$ es el área superficial y $l$ es el espesor de la pastilla, y tomando como factor geométrico, $g=\frac{l}{A}$, la ecuación (5) queda establecida como:

$$
\varepsilon^{\prime}=-\frac{g Z^{\prime \prime}}{\omega \varepsilon_{0}\left(Z^{\prime 2}+Z^{\prime \prime 2}\right)}
$$

La ecuación (6), el módulo de impedancia y el ángulo de fase se utilizaron para determinar la permitividad relativa $\varepsilon$ ' a diferentes temperaturas.

La Fig. 5 muestra los resultados de la permitividad en función de la temperatura, a diferentes frecuencias (medidas por espectroscopia de impedancia electroquímica) en el material KNL- $\mathrm{Nb}_{70} \mathrm{Ta}_{30} \mathrm{O}_{3}$. La temperatura de transición está en torno a $420{ }^{\circ} \mathrm{C}$, valor cercano al reportado por Zuo et al [4] y que es deseable en materiales de este tipo.

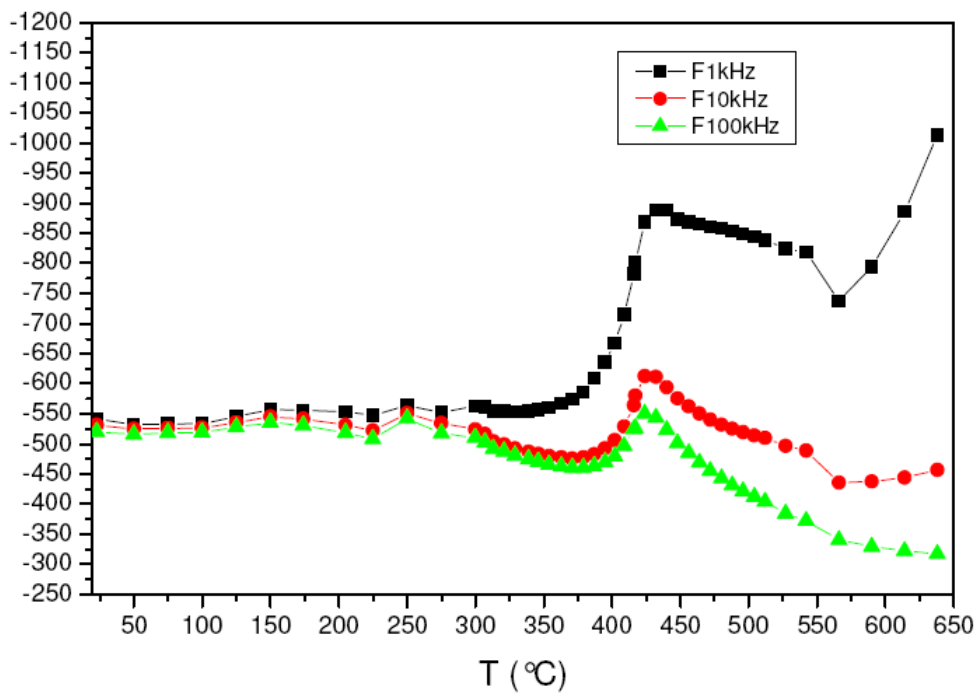

Figura 5. Temperatura vs. permitividad $\left(\varepsilon^{\prime}\right)$.

\section{Conclusiones}

Los materiales piezoeléctricos libres de plomo $\left(\mathrm{K}_{0,5} \mathrm{Na}_{0,5}\right)_{0,95} \mathrm{Li}_{0,05} \mathrm{Nb}_{\mathrm{y}} \mathrm{Ta}_{(1-\mathrm{y})} \mathrm{O}_{3}$ se obtuvieron sin que fuera necesario agregar un óxido como ayuda para lograr la sinterización del material. Estos materiales presentan una estructura ortorrómbica y el tamaño de grano parece no depender del contenido de tántalo, sino de las condiciones de procesamiento. La técnica de impedancia electroquímica ha sido útil en la determinación de la temperatura de transición, la cual es de $420{ }^{\circ} \mathrm{C}$, aproximadamente. Los resultados obtenidos indican que estos materiales se pueden utilizar como sustitutos del PZT. 


\section{Determinación de la Temperatura de Transición de Cerámicos Piezoeléctricos Libres de Plomo, Mediante Impedancia Electroquímica}

\section{Resumen}

Los materiales cerámicos piezoeléctricos con estructura perovskita basados en titanatos zirconato de plomo (PZT), presentan excelentes propiedades eléctricas cerca del límite de la fase morfotrópica (entre las fases romboédrica y tetragonal), lo que permite que sean ampliamente utilizados como actuadores, sensores y en dispositivos microelectrónicos. Sin embargo, la toxicidad del óxido de plomo $(\mathrm{PbO})$ y su alta presión de vapor durante el procesamiento, ha llevado a la búsqueda de materiales libres de plomo. Actualmente, las investigaciones de materiales piezoeléctricos libres de plomo se ha enfocado hacia niobatos alcalinos, titanatos de bismuto (BiT's) modificados, y sistemas en los que existe un límite de fase morfotrópica. Entre éstos, los niobatos alcalinos $(\mathrm{K}, \mathrm{Na}) \mathrm{NbO}_{3}(\mathrm{KNN})$ han sido estudiados con renovado interés, debido a que representan una alternativa menos contaminante para sustituir a los cerámicos piezoeléctricos base Plomo y presentan buenas propiedades piezoeléctricas y ferroeléctricas. En este trabajo, se sintetizaron cerámicos piezoeléctricos libres de plomo, basados en niobatos alcalinos, de acuerdo al sistema $\left(\mathrm{K}_{0,5} \mathrm{Na}_{0,5}\right)_{\mathrm{x}} \mathrm{Li}_{(1-\mathrm{x})} \mathrm{Nb}_{\mathrm{y}} \mathrm{Ta}_{(1-}$ y) $\mathrm{O}_{3}$, y se caracterizaron estructural y morfológicamente mediante difracción de rayos X y microscopía electrónica de barrido; para determinar su temperatura de transición, se utilizó espectroscopia de impedancia electroquímica, encontrándose que esta temperatura de transición está alrededor de $420{ }^{\circ} \mathrm{C}$, valor que es deseable para este tipo de materiales.

Palabras clave: PZT, niobatos alcalinos, temperatura de transición, cerámicos piezoeléctricos, cerámicos libres de plomo.

\section{Referencias}

1. Gene H. Haertling, "Ferroelectric Ceramics: History and Technology", $J$. Am. Cer. Soc. 82(4) (1999) 797-818.

2. T. Takenata and H. Nagata. "Present status of non-lead-based piezoelectric ceramics", Key Engineering Materials 157-158 (1999) 57-63.

3. Y. Li, W. Chen, Q. Xu, J. Zhou, X. Gu, S. Fang, "Electromechanical and dielectric properties of $\mathrm{Na}_{0.5} \mathrm{Bi}_{0.5} \mathrm{TiO}_{3}-\mathrm{K}_{0.5} \mathrm{Bi}_{0.5} \mathrm{TiO}_{3}-\mathrm{BaTiO}_{3}$ lead-free ceramics", Materials Chemistry and Physics 94 (2005) 328-332.

4. R. Zuo, J. Rödel, R. Chen, L. Li, "Sintering and electrical properties of lead-free $\mathrm{Na}_{0.5} \mathrm{~K}_{0.5} \mathrm{NbO}_{3}$ ", J. Am. Ceram. Soc. 89 (2006) 2010-2015.

5. M. Matsubara, K. Kikuta, S. Hirano, "Piezoelectric properties of $\left(\mathrm{Na}_{0.5} \mathrm{~K}_{0.5}\right)\left(\mathrm{Nb}_{1-\mathrm{x}} \mathrm{Ta}_{\mathrm{x}}\right) \mathrm{O}_{3}-\mathrm{K}_{5.4} \mathrm{CuTa}_{10} \mathrm{O}_{29}$ ceramics", J. Appl. Phys. 97 (2005) 114105. 